Review

\title{
Early Diagnosis of Prostate Cancer from the Perspective of Chinese Physicians
}

\author{
Wei Yu, Liqun Zhou ${ }^{凶}$ \\ Department of Urology, Peking University First Hospital, Institute of Urology, Peking University, National Urological Cancer Center of China, Beijing, China \\ $\square$ Corresponding author: Liqun Zhou, Department of Urology, Peking University First Hospital, Institute of Urology, Peking University, National Urological Cancer \\ Center of China, Beijing 100034, China. Telephone: +86-10-83575006; Fax: +86-10-66551726; Email: zhoulqmail@sina.com \\ () The author(s). This is an open access article distributed under the terms of the Creative Commons Attribution License (https://creativecommons.org/licenses/by/4.0/). \\ See http://ivyspring.com/terms for full terms and conditions.
}

Received: 2019.05.15; Accepted: 2020.01.06; Published: 2020.03.05

\begin{abstract}
Prostate cancer $(\mathrm{PCa})$ is the seventh most diagnosed cancer and the tenth leading cause of cancer mortality in China. Unlike the USA, both incidence and mortality continue to increase. In China, PCa is often diagnosed at a locally advanced or metastatic stage, resulting in a high mortality-to-incidence ratio. Implementing regular screening using a well-validated biomarker may result in the earlier diagnosis of localized disease. Furthermore, it is important to be able to distinguish between low-grade and high-grade disease, to avoid subjecting patients to unnecessary biopsies, undertreatment of significant disease, or overtreatment of indolent disease. While prostate-specific antigen (PSA) is commonly used in PCa screening around the world, its relationship to $\mathrm{PCa}$ is still unclear and results vary widely across different studies. New biomarkers, imaging techniques and risk predictive models have been developed in recent years to improve upon the accurate detection of high-grade PCa. Blood- and urine-based biomarkers, such as PSA isoforms, prostate cancer antigen 3, or mRNA transcripts, have been used to improve the detection of high-grade PCa. These markers have also been used to create risk predictive models, which can further improve PCa detection. Furthermore, multiparametric magnetic resonance imaging is becoming increasingly accessible for the detection of PCa. Because of ethnic variations, biomarkers and risk predictive models validated in Western populations cannot be directly applied to Chinese men. Validation of new biomarkers and risk predictive models in the Chinese population may improve $\mathrm{PCa}$ screening and reduce mortality of this disease in China.
\end{abstract}

Key words: prostate cancer, biomarkers, risk predictive models

\section{Introduction}

In 2018, prostate cancer (PCa) was estimated to have the second highest incidence of cancers globally, contributing to $3.8 \%$ of deaths from cancer [1]. In China, PCa was estimated to be the seventh most commonly diagnosed cancer and the tenth leading cause of cancer mortality in men [2,3]. Unlike the US, which has seen a decrease in incidence and mortality, both incidence and mortality are increasing in China. [2,4] The difference in mortality trends may be attributed to the establishment of regular $\mathrm{PCa}$ screening in the US, resulting in most incidences of PCa being diagnosed when the disease is localized [4]. In China, PCa is often diagnosed at a locally advanced or metastatic stage, which may contribute to the high mortality-to-incidence ratio, close to $50 \%$ [2]. This may be due to the lack of mandatory or regular screening in China, as well as a lack of well-established biomarkers or screening methods in the Chinese population. Implementation of regular screening may result in earlier diagnosis of localized disease.

Prostate-specific antigen (PSA) is a marker related to the early diagnosis of PCa and is used for PCa screening around the world. While positive biopsy results correlate with elevated serum levels of PSA, the relationship between PSA and PCa varies greatly between different studies [5,6]. Furthermore, the use of total PSA levels as a diagnostic decision-making tool for biopsies has a high rate of both false-negative and false-positive results, leading to delayed diagnoses as well as unnecessary biopsies. 
Thus, using PSA alone can lead to over-diagnosis [7]. Multiparametric magnetic resonance imaging (mpMRI) may also be used prior to transrectal ultrasound (TRUS)-guided biopsy. However, while mpMRI has a high sensitivity for $\mathrm{PCa}$, it is less specific than both biopsy and PSA [8]. As such, the usefulness of mpMRI alone in the early diagnosis of PCa is limited.

This situation emphasizes the need for effective biomarkers and imaging techniques which, when combined with clinical factors, can help establish effective risk predictive models to improve earlier diagnosis of PCa. This review provides an update on recent developments in $\mathrm{PCa}$ diagnostics, screening tools and risk predictive models, and their applicability to the Chinese population.

\section{The value of new biomarkers in the early diagnosis of prostate cancer}

TRUS-guided biopsies are required for the diagnosis of $\mathrm{PCa}$, but carry a risk of adverse events, including sepsis [9]. Furthermore, biopsies can identify the presence of low-grade tumors that pose minimal risk of progression, resulting in overtreatment of the patient. An accurate pre-biopsy triage of patients who are suspected of having $\mathrm{PCa}$ would reduce the number of unnecessary biopsies, avoiding the pain and the risk of other adverse events associated with biopsies. Additionally, a minimally invasive prebiopsy test could be carried out more frequently for screening purposes, allowing earlier detection of aggressive disease.

\section{Prostate cancer antigen 3 (PCA3)}

PCA3 is an overexpressed non-coding RNA in $\mathrm{PCa}$, but undetectable in normal tissue or other tumor types [10]. The PCA3 score is calculated as a ratio of PCA3 mRNA/PSA mRNA and has been demonstrated to be highly accurate in predicting $\mathrm{PCa}$, more so than PSA alone [11,12]. The US Food and Drug Administration (FDA) approved the use of a PCA3 test for the detection of PCa, using a PCA3 score cut-off of 25 to determine the likelihood of a positive diagnosis [13]. Although the optimal cut-off score is still subject to debate, an increasing PCA3 score strongly correlates with a higher probability of PCa overall, as well as high-grade PCa [12,14]. In a Chinese study, a decision curve analysis indicated that a PCA3-based model had a superior net benefit at almost all threshold probabilities compared with percentage-free PSA (\%fPSA) or PSA density (PSAD)[15].

\section{Prostate Health Index (PHI)}

The PHI is a risk score calculated using a combination of PSA isoforms, [-2]proPSA (p2PSA), $\%$ fPSA and total PSA (tPSA), each of which improves prediction of PCa compared with PSA alone.[16] PHI was found to be significantly more accurate in predicting the presence of PCa in men with negative digital rectal examinations (DRE) and a tPSA of 2-10 $\mathrm{ng} / \mathrm{mL}$ than with tPSA alone, which could not differentiate between individuals with or without PCa.[17] More importantly, while PCA3 was more accurate at predicting PCa overall, PHI was the most accurate predictor of significant PCa when compared with PCA3, tPSA, or PSAD, and correlated well with the Gleason Score $[16,18,19]$. This was true in both European and Chinese populations $[18,20]$. The addition of age, DRE and/or prostate volume (PV) to the PHI did not increase its predictive power in identifying high-grade PCa in Chinese men.[20] PHI may increase the ability to detect $\mathrm{PCa}$ while decreasing the number of unnecessary biopsies by almost $50 \%$; only a few cases of clinically significant PCa are likely to be missed using this index [19]. PSA can sometimes be elevated above $10 \mathrm{ng} / \mathrm{mL}$ for benign tissue, which can cause a false-positive result; however, the PHI was demonstrated to accurately identify the presence of high-grade PCa even in men with significantly elevated serum PSA [19]. The PHI has been approved by the FDA for the detection of PCa in men aged 50 years and older and a tPSA level of $4-10 \mathrm{ng} / \mathrm{mL}$ and negative DRE [21].

\section{Kscore ${ }^{\circledR}$}

4 Kscore ${ }^{\circledR}$ (OPKO Health, Miami, USA) is a commercially available PCa testing kit, using a combination of tPSA, \%fPSA, intact PSA and human Kallikrein 2 (hK2) with age, DRE results and history of prior biopsies [22]. This test is able to distinguish between the presence and absence of high-grade $\mathrm{PCa}$, as well as identifying patients with tumors that are likely to progress. The test also increases the predictive discrimination for 15-20-year risk of PCa mortality among men with elevated PSA $[22,23]$. The 4 Kscore ${ }^{\circledR}$ can be used to reduce the number of unnecessary biopsies by over $30 \%$ in men with elevated PSA, with little risk of missing high-grade disease [24,25]. As of December 2018, the 4Kscore ${ }^{\circledR}$ test was not FDA-approved, but is offered as a Laboratory Developed Test through certified and accredited laboratories and wholly owned subsidiaries of OPKO Health.

\section{TMPRSS2: ERG}

In addition to serum biomarkers, urinary biomarkers are also of increasing interest. Fusion of the TMPRSS2 (transmembrane protease, serine 2) and ERG (a member of the E26 transformation-specific family of oncogenes) genes is highly specific for $\mathrm{PCa}$, as well as displaying $93.2 \%$ specificity for predicting 
clinically significant PCa.[12,26] This gene fusion has been found in approximately $50 \%$ of PCa patients, and fused RNA transcripts can be detected in the urine, providing the added benefit of being a non-invasive test [27]. TMPRSS2:ERG mRNA is elevated in men with $\mathrm{PCa}$, and is associated with tumors with a high Gleason Score [28]. TMPRSS2:ERG also correlated with tumor dimensions at the time of prostatectomy, and this urine test had a greater area under the receiver operating characteristic (ROC) curve (AUC) than serum PSA for the detection of PCa [28]. However, ERG nuclear expression (which is correlated to TMPRSS2:ERG rearrangement) is significantly lower in PCa samples for men of Chinese origin than samples from the UK, suggesting that TMPRSS2:ERG rearrangement may not be a suitable biomarker for detecting PCa in Chinese men.[29] Currently, the use of this biomarker has not been approved by the FDA.

\section{SelectMDx® and ConfirmMDx®}

SelectMDx (MDxHealth, Irvine, USA) is a commercially available, urine-based test for PCa. DLX1 and HOX6 expression is associated with an increased likelihood of high-grade $\mathrm{PCa}$, and mRNA transcripts of these two genes can be detected in urine [30]. The combination of these two mRNA markers with traditional clinical parameters, including PSA, PSAD, DRE, age and family history, is used in the SelectMDx test [31]. Another test developed by MDxHealth is ConfirmMDx, which uses residual cancer-negative prostate biopsy samples. The basis of ConfirmMDx is the 'field effect', whereby molecular changes, such as epigenetic changes, occur in benign-looking tissue adjacent to cancerous cells [32]. These changes cannot be detected by histopathology, but can be detected using methylation-specific polymerase chain reaction (MSP). Abnormal methylation of genes such as GSTP1, APC and RASSF1 is often detected in PCa. ConfirmMDx uses multiplex MSP to determine the epigenetic status of these genes in histopathologically negative tissue, and aids the detection of occult PCa [33]. To date, neither SelectMDx nor ConfirmMDx has obtained FDA approval.

While the biomarkers outlined above and screening techniques are improving the accuracy of early diagnosis, each technique has its limitations, calling for the need to develop better diagnostic methods/models to improve the early detection of PCa.

\section{The value of mpMRI in the early diagnosis of prostate cancer}

mpMRI may also be widely used as a triage test prior to TRUS-guided biopsy. The PROMIS study evaluated mpMRI in men with elevated PSA levels, followed by TRUS-guided biopsy and template prostate mapping (TPM) biopsy [9]. Of the three techniques used, mpMRI was the most sensitive in detecting clinically significant $\mathrm{PCa}$, and patients negative for mpMRI may be able to avoid unnecessary biopsies [8,9]. However, mpMRI was less specific than both biopsy techniques, with a high false-positive rate; therefore, patients positive for $\mathrm{PCa}$ by mpMRI still require a biopsy to confirm the presence of clinically significant disease prior to treatment $[8,9]$. Because mpMRI is a subjective test, there is a risk of interobserver variability; the effectiveness of mpMRI depends on the skill of the technician performing the analysis, and can lead to reproducibility issues [34]. A MRI suspicion score (mSS), utilizing MRI-ultrasound fusion targeted prostate biopsy (MRF-TB), was used by Meng et al. to provide a grading of cancer suspicion and predict the likelihood of aggressive disease [35]. While overall detection of PCa was not significantly different to standard TRUS-guided biopsies, MRF-TB was superior in detecting high-grade $\mathrm{PCa}$, while also noticing fewer low-grade PCa in men who had not previously undergone prostate biopsies [35,36]. Furthermore, a higher mSS score was associated with a higher detection rate of high-grade tumors [35]. In future, lowering costs of mpMRI and other imaging techniques mean that these techniques will become more accessible. If mpMRI can be validated in different populations, and as it becomes widely available, it will be an invaluable tool in the early detection of significant tumors, helping to limit unnecessary biopsies in men with no or indolent disease.

Imaging techniques, such as mpMRI, are improving the accuracy of early diagnosis, but each individual technique has its limitations. Risk predictive models combining multiple variables to determine the possibility of PCa may be able to further improve the early diagnosis of PCa.

\section{The value of risk predictive models in the early diagnosis of prostate cancer}

Given that using individual biomarkers may not always be accurate, combining multiple variables has been trialled to reduce the limitations of each technique and increase predictive ability. The variables used in these risk calculators are detailed in Table 1.

Two particularly prominent risk calculators have been developed from large PCa screening trials in the US and Europe: the Prostate Cancer Prevention Trial Risk Calculator (PCPT-RC) and the European Randomized Study of Screening for Prostate Cancer Risk Calculator (ERSPC-RC). 
Table 1. Variables included in prostate cancer (PCa) diagnostic tests.

\begin{tabular}{|c|c|c|c|c|c|c|c|c|c|c|c|c|c|}
\hline PCa diagnostic test & Age & Race & $\begin{array}{l}\text { Family } \\
\text { history }\end{array}$ & DRE & $\begin{array}{l}\text { Prostate } \\
\text { volume }\end{array}$ & $\begin{array}{l}\text { Previous } \\
\text { biopsy }\end{array}$ & TRUS & PSA & PCA3 & fPSA & PHI & mpMRI & $\begin{array}{l}\text { Other } \\
\text { markers }\end{array}$ \\
\hline PHI[16] & & & & & & & & $\bullet$ & & $\bullet$ & & & $\bullet$ a \\
\hline 4 Kscore ${ }^{\circledR}[23]$ & $\bullet$ & & & $\bullet$ & & $\bullet$ & & $\bullet$ & & $\bullet$ & & & $\bullet$ b \\
\hline РСРТ[38] & $\bullet$ & $\bullet$ & $\bullet$ & $\bullet$ & & $\bullet$ & & $\bullet$ & & & & & \\
\hline PCPT 2.0[39] & $\bullet$ & $\bullet$ & $\bullet$ & $\bullet$ & & $\bullet$ & & $\bullet$ & & $\bullet$ & & & \\
\hline PCPT-PHI[44] & $\bullet$ & $\bullet$ & $\bullet$ & $\bullet$ & & $\bullet$ & & $\bullet$ & & $\bullet$ & $\bullet$ & & \\
\hline РСРТ-РСА3[14] & $\bullet$ & $\bullet$ & $\bullet$ & $\bullet$ & & $\bullet$ & & $\bullet$ & $\bullet$ & & & & \\
\hline ERSPC C [40] & $\bullet$ & & $\bullet$ & $\bullet$ & & $\bullet$ & $\bullet$ & $\bullet$ & & & & & \\
\hline ERSPC-PHI[44] & $\bullet$ & & $\bullet$ & $\bullet$ & $\bullet$ & $\bullet$ & & $\bullet$ & & & $\bullet$ & & \\
\hline Loeb et al.[44] & $\bullet$ & & & & $\bullet$ & $\bullet$ & & $\bullet$ & & & $\bullet$ & & \\
\hline STHLM3 study[45] & $\bullet$ & & $\bullet$ & $\bullet$ & $\bullet$ & $\bullet$ & & $\bullet$ & & $\bullet$ & & & $\bullet d$ \\
\hline MiPS[48] & & & & & & & & $\bullet$ & $\bullet$ & & & & $\bullet$ \\
\hline Radtke et al.[51] & $\bullet$ & & & $\bullet$ & $\bullet$ & & & $\bullet$ & & & & $\bullet$ & \\
\hline Cao et al.[57] & & & & & & & & & $\bullet$ & & & & $\bullet$ f \\
\hline PHI-nomogram[58] & $\bullet$ & & & & $\bullet$ & & & & & & $\bullet$ & & \\
\hline Chiu et al.[20] & $\bullet$ & & & & $\bullet$ & & & & & & $\bullet$ & & \\
\hline Huashan RC1[59] & $\bullet$ & & & $\bullet$ & $\bullet$ & & $\bullet$ & $\bullet$ & & $\bullet$ & & & \\
\hline Huashan RC2[59] & $\bullet$ & & & $\bullet$ & & & & $\bullet \mathrm{g}$ & & $\bullet$ & & & \\
\hline PCP[15] & & & & & & & & $\bullet$ & & $\bullet$ & & & $\bullet$ h \\
\hline CPCC-RC[60] & $\bullet$ & & & $\bullet$ & $\bullet$ & & & $\bullet$ & & $\bullet$ & & & \\
\hline Niu et al.[61] & $\bullet$ & & & & & & & $\bullet$ i & & & & $\bullet$ & \\
\hline van Leeuwen et al.[52] & $\bullet$ & & & $\bullet$ & & & & $\bullet$ & & & & $\bullet$ & \\
\hline PBRS[53] & $\bullet$ & & & & $\bullet$ & & & $\bullet j$ & & & & $\bullet$ & \\
\hline
\end{tabular}

aPHI also used p2PSA in its calculations. bOther markers in the 4Kscore ${ }^{\circledR}$ test include intact PSA and hK2. cThe ERSPC comprises multiple risk calculators using different combinations of variables. dOther markers included in the STHLM3 test include intact PSA, hK2, MSMB, MIC1 and genetic markers. eUrinary levels of TMPRSS2:ERG, Annexin A3 and Sarcosine were also included. fTMPRSS2:ERG score was used in the MiPS. gLogPSA was used for both Huashan risk calculators. hPCP risk calculator included complexed PSA in its calculations. TThe nomogram developed by Niu et al. included adjusted PSA density. iThe PBRS utilised both PSA and PSA density. CPCC-RC: Chinese Prostate Cancer Consortium Risk Calculator; DRE: digital rectal examination; ERSPC: European Randomized Study of Screening for Prostate Cancer; fPSA: free PSA; hK2: human kallikrein 2; MIC1: macrophage inhibitory cytokine 1; MiPS: Mi-Prostate score; MSMB: microseminoprotein-beta; mpMRI: multiparametric magnetic resonance imaging; p2PSA: [-2]proPSA; PBRS: prostate biopsy rating scale; PCA3: prostate cancer antigen 3; PCP: Prostate Cancer Predictor; PCPT: Prostate Cancer Prevention Trial; PHI: Prostate Health Index; PSA: prostate-specific antigen;STHLM3: Stockholm 3; TRUS: transrectal ultrasound.

\section{Prostate Cancer Prevention Trial Risk Calculator (PCPT-RC)}

The PCPT-RC was developed with a cohort of 5,519 men over the age of 55 years with a PSA value less than $3.0 \mathrm{ng} / \mathrm{mL}$. This risk calculator is available for use online and takes into account race, age, DRE, PSA, family history and prior biopsy results [37]. While the PCPT-RC score correlated with the risk of $\mathrm{PCa}$, the ROC curve for the risk calculator was not statistically significantly different to PSA alone, with an exception for African-American men, where the PCPT-RC outperformed PSA alone [38]. In 2014 PCPT-RC 2.0 was released, which included the \%fPSA into the calculator. Although this improved the identification of significant disease, it was not able to distinguish between high- and low-grade diseases, or low-grade disease and absence of disease [39].

\section{European Randomized Study of Screening for Prostate Cancer Risk Calculator (ERSPC-RC)}

ERSPC-RC is a series of multistep risk calculators that can be used at various stages of screening and does not require blood tests [40]. ERSPC developed six risk calculators, which were dependent on the information available, such as PSA, PHI or Gleason Score. The newest of the risk calculators, ERSPC-RC6, can be used to determine the likelihood of PCa for the proceeding four years [40]. After 13 years of follow-up, the ERSPC-RC was able to reduce unnecessary biopsies by reducing the number of screenings needed to prevent one PCa death, from 1,055 to 781 men [41]. The ERSPC-RC is significantly better at predicting PCa overall and specifically for high-grade PCa than the PCPT-RC (AUC: 0.741 [95\% CI, 0.717-0.763] vs 0.692 [95\% CI, 0.668-0.716], $P<$ 0.001) [42]. However, the ERSPC-RC tended to under-predict the presence of $\mathrm{PCa}$, resulting in men with PCa who were left untreated [42].

The ability to detect the presence of PCa was not significantly different between ERSPC-RC and PCPT-RC 2.0; however, in detecting significant PCa, the AUC for the ERSPC-RC was significantly higher than for the PCPT-RC 2.0 (0.73 vs 0.70; DeLong test, $P$ $=0.043$ ) [43]. Decision curve analyses indicated that both risk calculators provide a clinical net benefit in the threshold probability range, between $18 \%$ and $40 \%$ for any PCa and between $8 \%$ and $40 \%$ for clinically significant PCa $[42,43]$.

\section{Predictive models combining new biomarkers can improve early diagnosis of prostate cancer}

To increase the net benefit from these risk calculators, other biomarkers have been included with these risk predictive models. Loeb et al. investigated the value of adding PHI to the PCPT-RC or ERSPC-RC in a population of 728 men with PSA levels of $2-10 \mathrm{ng} / \mathrm{mL}$ and negative DRE [43]. Discrimination of aggressive disease was significantly improved by 
the addition of PHI to both risk calculators, and a new model was designed including age, previous biopsy, PV, PSA and PHI for the prediction of significant PCa. This new model offered further improvement to the PCPT-RC or ERSPC-RC, and showed a net benefit for the model at threshold values greater than 3\% [44]. Similarly, the addition of PCA3 or TMPRSS:ERG to PCPT-RC or ERSPC-RC also improved the predictive value of these risk calculators $[12,14,28]$.

The Stockholm 3 (STHLM3) study, established in Sweden, utilized a combination of plasma protein biomarkers (PSA, fPSA, intact PSA, hK2, microseminoprotein-beta [MSMB] and macrophage inhibitory cytokine 1 [MIC1]), genetic markers (based on single nucleotide morphisms of 254 genes, including HOXB13), clinical variables (age, family history and previous biopsy) and prostate exam results (DRE and PV) to develop a risk predictive model $[45,46]$. When STHLM3 was compared with the PSA test alone, at the same sensitivity, it significantly improved the specificity of PCa detection and was able to reduce the number of unnecessary biopsies by $44 \%[45,47]$.

The Mi-Prostate score (MiPS) combines PCA3 and TMPRSS:ERG scores with PSA levels to ascertain the risk of PCa [48]. Addition of the TMPRSS:ERG score to PCA3 and total PSA significantly improved the AUC compared with PCA3 plus PSA or TMPRSS:ERG plus PSA [48]. Addition of MiPS to the PCPT-RC increased the net benefit of the model and further reduced unnecessary biopsies across all threshold probabilities for PCa, and specifically high-grade PCa [48]. However, this is the only study using the MiPS score with the PCPT-RC, and will require further validation in more studies and patient populations before widespread use as a risk predictive model for PCa.

\section{Including mpMRI in predictive models can improve the early diagnosis of prostate cancer}

In addition to the incorporation of new biomarkers into risk predictive models, mpMRI has also been used to enhance the predictive power of these models. The addition of the Prostate Imaging Reporting and Data System (PI-RADS) score, an evaluation score based on mpMRI, to a risk calculator with age, PSA, and DRE-PV significantly increased the AUC, compared with risk calculators without mpMRI $[49,50]$. Inclusion of mpMRI into the risk calculation also reduced the number of unnecessary biopsies, while missing few high-grade PCa. A risk model developed by Radtke et al. incorporated PSA, PV, DRE, age, and the PI-RADS score to determine the risk of significant PCa [51]. Two separate nomograms were developed for men who were biopsy-naïve and those who had a previous biopsy. The risk model was comparable to that of ERSPC-RC3 (for biopsy-naïve men) with PI-RADS, while in men with a previous biopsy the discrimination of this risk model was better than that of ERSPC-RC4 (for men with previous biopsies) with or without a PI-RADS score. Both risk models used by Radtke et al. produced a higher net benefit compared with the ERSPC-RCs in terms of detecting patients with significant $\mathrm{PCa}$, and may provide a clinically useful tool for men in whom biopsy is being considered [51]. van Leeuwen et al. further incorporated previous biopsy into a risk model with the same parameters used by Radtke et al. [52]. The inclusion of the PI-RADS score into the risk model significantly improved prediction of significant PCa compared to risk models excluding PI-RADS [52].

A recent study investigated the use of the PI-RADS version 2 score with age, PSA, PSAD and PV to calculate the prostate biopsy rating scale (PBRS) in Chinese men [53]. The PBRS score was found to reduce the number of unnecessary biopsies by up to $63 \%$, when compared to using PSA alone, which is the current standard of PCa detection in China. [53] The PBRS also demonstrated improvements when compared to the biomarkers alone in accurately in the AUC curve for all PCa, PCa with a Gleason score $\geq 7$, $\mathrm{PCa}$ with a clinical stage $\geq \mathrm{T} 2 \mathrm{~b}$, and $\mathrm{PCa}$ with D'Amico risk > low. However, this was a retrospective analysis and many men with PSA levels of 4$10 \mathrm{ng} / \mathrm{ml}$ refused biopsies and opted for follow-up observation and were underrepresented in this study. As such, while the PBRS shows promise, further investigation is required to validate this model [53].

\section{Predictive models that combine new biomarkers and mpMRI may improve early diagnosis of prostate cancer}

New biomarkers and mpMRI have improved the accuracy of PCa risk prediction compared with more traditional parameters, such as tPSA and DRE. The use of new biomarkers and mpMRI together have the potential to further improve risk prediction. The combination of mpMRI and PCA3 improved the accuracy of predicting prostate biopsy outcomes when compared with PCA3 alone (0.726 vs 0.750 ) [54]. Furthermore, the addition of mpMRI to PCA3 testing also improved sensitivity ( 0.793 vs 0.680$)$, positive and negative predictive values and the AUC (0.857 vs $0.825 ; P<0.001)$ [54]. When mpMRI or a new biomarker (PHI and PCA3) was added to a base model of DRE and age, mpMRI improved risk prediction, but not with biomarkers alone [55]. In this study, the further addition of PHI or PCA3 to the mpMRI model did not provide further benefit in ROC or decision curve analyses [55]. This was corroborated 
by Gnanapragasam et al., who also found that the addition of PSA or PHI to mpMRI results did not generate a clinically relevant benefit [56]. However, the addition of PHI (at a cut-off $\geq 35$ ) was able to significantly improve the predictive performance for detecting clinically significant cancers (negative predictive value of 0.97 and sensitivity of 0.99 ) [56]. Furthermore, PHI was able to predict the presence of significant PCa in men with negative mpMRI, reducing the likelihood of missed PCa [56].
The inclusion of clinical factors, such as new biomarkers and/or mpMRI, to risk calculators increases the ability to distinguish high-grade and low-grade $\mathrm{PCa}$, compared with risk calculators without clinical factors, or clinical factors alone. These non-invasive and minimally invasive tests can reduce the number of unnecessary biopsies and overtreatment, while still identifying patients who require aggressive therapy. The AUC for these biomarkers and risk predictive models is summarized in Table 2.

Table 2. Prevalence of prostate cancer and area under the ROC curve for biomarkers and risk predictive models

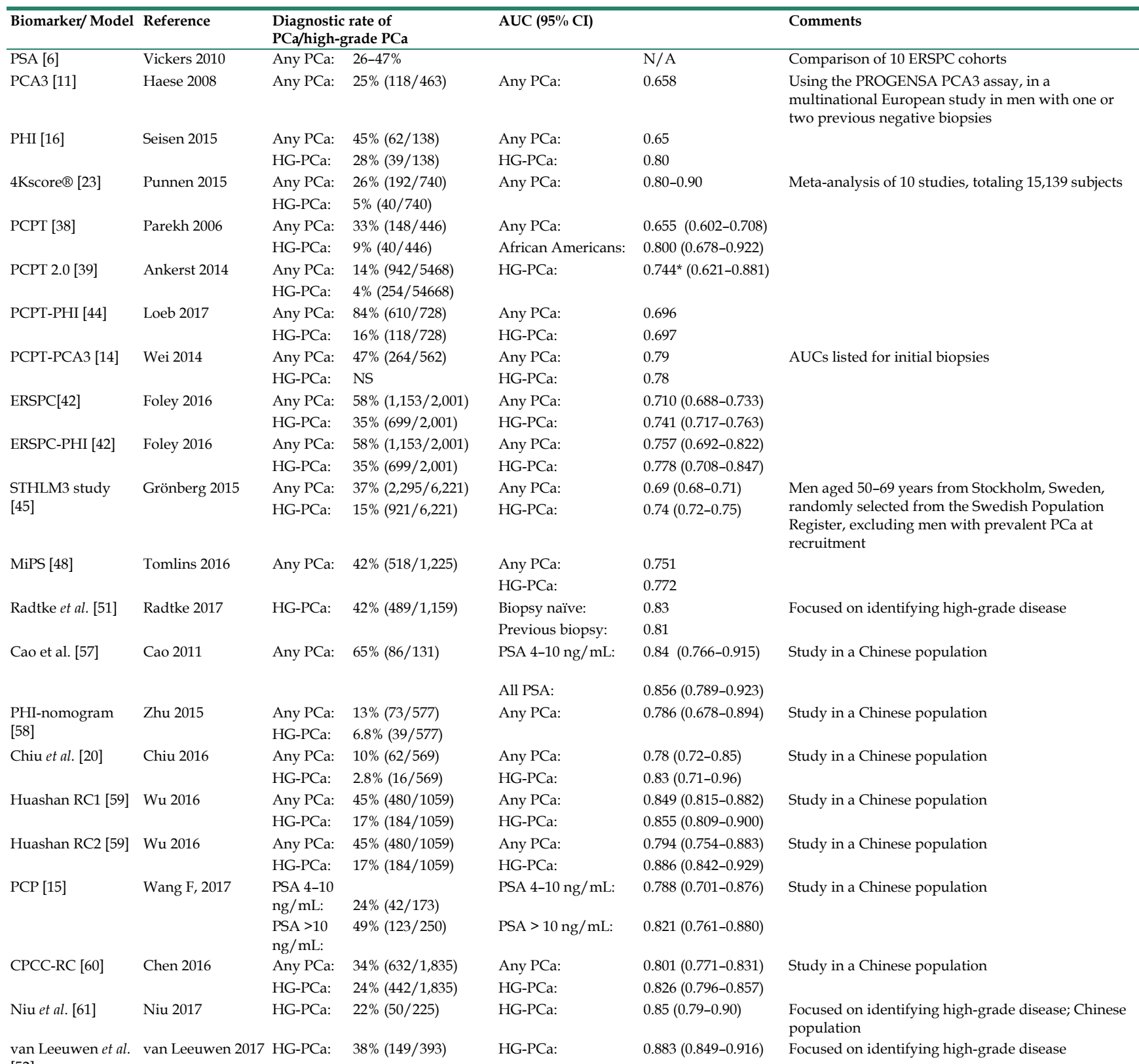
$[52$

*Median AUC (range). AUC: area under the receiver operating characteristic curve; CI: confidence interval; CPCC-RC: Chinese Prostate Cancer Consortium Risk Calculator; ERSPC: European Randomized Study of Screening for Prostate Cancer; HG-PCa: high-grade prostate cancer; MiPS: Mi-Prostate score; PCA3: prostate cancer antigen 3; PCP: Prostate Cancer Predictor; PCPT: Prostate Cancer Prevention Trial; PHI: Prostate Health Index; PSA: prostate-specific antigen;STHLM3: Stockholm 3. 


\section{Risk predictive models for Chinese populations}

While the PCPT-RC and ERSPC-RC fit the European and American populations, they have not been tested in Chinese cohorts, and ethnic variations suggest that they may not be as reliable. To rectify this, studies have been conducted to develop PCa risk calculators that are optimized for the Chinese population. A multiplex model using urinary PCA3, TMPRSS:ERG, Annexin A3 and Sarcosine was developed by Cao et al., and was found to perform significantly better than PSA alone in predicting PCa in Chinese men [57]. However, this study did not distinguish between the identification of low- and high-grade disease; thus, it would not be useful in the reduction of unnecessary biopsies [57]. On the other hand, a PHI-based nomogram developed by Zhu et al. using PHI, age and PV was effective in predicting $\mathrm{PCa}$, and would reduce the number of unnecessary biopsies by $27 \%$ [58]. A separate study by Chiu et al. also used PHI, age, and PV to develop a risk calculator in a Chinese cohort and was able to reduce more unnecessary biopsies than PSA alone, PSA, DRE-PV with age, or PHI alone at all risk thresholds; $80.2 \%$ of unnecessary biopsies could be avoided at the $20 \%$ risk threshold for high-grade PCa [20]. This risk calculator was also able to reduce the number of biopsies when specifically looking for high-grade disease.

The Huashan risk calculators developed by $\mathrm{Wu}$ et al. were better able to identify Han Chinese patients with PCa than the PCPT-RC, using age, DRE result, PV, PSA, \%fPSA and TRUS-guided biopsy results (Huashan RC1)[59]. In particular, the Huashan RC1 had a greater AUC compared with PCPT-RC and the Huashan RC2, and thus able to reduce the greatest number of unnecessary biopsies. Another model, the Prostate Cancer Predictor (PCP), used a combination of tPSA, fPSA, and complexed PSA (cPSA) to develop a model that was strongly correlated with PCa [49]. PCP had a greater specificity for PCa than tPSA, fPSA, $\%$ fPSA, or cPSA alone and was able to reduce the number of unnecessary biopsies $(22.8 \%$ vs $11.1 \%$, $11.2 \%, 17.4 \%$ and $15.5 \%$, respectively) [49]. A study in Shanghai established the Chinese Prostate Cancer Consortium Risk Calculator (CPCC-RC), which incorporated age, $\log \mathrm{PSA}, \log \mathrm{PV}, \mathrm{fPSA}$ and DRE to calculate the risk of $\mathrm{PCa}$ (model 1) or high-grade $\mathrm{PCa}$ (model 2) [60]. The CPCC-RC showed better accuracy and clinical benefit in the Chinese population than either the ERSPC-RC or PCPT-RC, with an AUC of 0.801 (95\% CI, 0.771-0.831) and 0.826 (95\% CI, 0.7960.857 ) for models 1 and 2, respectively [60].

As the use of mpMRI becomes more common in China, PI-RADS scores can be incorporated into risk predictive models. Niu et al. developed a nomogram (LR model) for the detection of high-grade PCa in men with PSA levels between 4 and $10 \mathrm{ng} / \mathrm{mL}$ [61]. This model, which incorporates age, adjusted-PSAD and PI-RADS v2, had an AUC of 0.85 (95\% CI, 0.790.90 ), sensitivity of $87.3 \%$, and specificity of $78.4 \%$ at a threshold risk score $\geq 0.36$ [61]. Although no decision curve analyses were performed, the high performance of the LR model suggests that it is an effective tool in identifying high-grade PCa in Chinese men with PSA levels in the "gray zone" and, therefore, has the potential to reduce unnecessary biopsies [61].

Thus, as outlined above, many risk predictive models are well established in European and American populations. However, when these models were applied to Chinese populations, the results were less than optimal. Several models have now been developed in Chinese cohorts. Although these models still require further validation, they may better assist in the early detection of high-grade PCa in Chinese men.

\section{Future directions}

Current risk calculators are more accurate than clinical factors (either biomarkers or mpMRI) individually, but there is still room for improvement. There are currently more than 100 ongoing studies registered on ClinicalTrials.gov investigating methods of detection or diagnosis of $\mathrm{PCa}$, two of which are recruiting in China (Table 3). A further three studies involve the validation of risk predictive models and includes the use of MRI (Table 4). Other studies explore the use of biparametric MRI, positron emission tomography (PET)/computed tomography (CT) scans, including the use of radiolabeling with fludeoxyglucose $\left({ }^{18} \mathrm{~F}\right)$ or Gallium-68, for the detection of PCa. Eleven of the ongoing studies are currently in Phase III or IV validation (Table 5).

The subjective nature of mpMRI for diagnosis is a significant limitation to the technique, as well as the time-consuming nature of image analysis. However, this may be overcome in the future through computer-aided detection, which has been relatively successful in the analysis of mammograms in breast cancer, as well as CT colonography. Over the past decade, many studies were performed in patients suspected of PCa, but most of these had small sample sizes with fewer than 100 participants (summarized by Afef et al.) [62]. Computer-aided detection and/or diagnosis can remove interoperator variability, provided a single, optimized algorithm is used, as different systems will have differing levels of accuracy [62]. The algorithm developed by Roethke et al. was comparable in accuracy to human analyses, although this study used a small sample size and the older version of the PI-RADS scoring system [63]. 
Table 3. Ongoing studies currently recruiting in China investigating the diagnosis or detection of prostate cancer.

\begin{tabular}{|c|c|c|c|c|c|}
\hline Study title & NCT number & Intervention & Geographic location & Number enrolled & Study start \\
\hline $\begin{array}{l}\text { Establishment and clinical assessment of a prostate cancer risk model based } \\
\text { on the updated circulating tumor cell detection technique }\end{array}$ & NCT02940977 & $\begin{array}{l}\text { Other: blood } \\
\text { draws }\end{array}$ & Shanghai, China & 120 & October 2016 \\
\hline $\begin{array}{l}\text { ICG-based fluorescence imaging in localization of prostate cancer and } \\
\text { metastatic lymph nodes }\end{array}$ & NCT02840617 & Drug: ICG & Guangdong, China & 50 & March 2016 \\
\hline
\end{tabular}

ICG: indocyanine green.

Table 4. Current ongoing studies involving risk predictive models.

\begin{tabular}{|c|c|c|c|c|c|}
\hline Study title & NCT number & Biomarkers and/or mpMRI & Geographic location & Number enrolled & Study start \\
\hline $\begin{array}{l}\text { Early and accurate detection of prostate } \\
\text { cancer in general practice }\end{array}$ & NCT03431753 & $\begin{array}{l}\text { STHLM3 + mpMRI } \\
\text { PSA + mpMRI }\end{array}$ & $\begin{array}{l}\text { Denmark } \\
\text { Sweden }\end{array}$ & 3,000 & February 2018 \\
\hline $\begin{array}{l}\text { MRI and biomarkers in prostate cancer } \\
\text { (Multi-IMPROD) }\end{array}$ & NCT02241122 & $\begin{array}{l}\text { MRI } \\
\text { Serum biomarkers } \\
\text { Urine biomarkers }\end{array}$ & Finland & 400 & September 2014 \\
\hline $\begin{array}{l}\text { Improved prostate cancer diagnosis - } \\
\text { combination of rapid prebiopsy MRI and } \\
\text { biomarkers (IMPROD2_0) }\end{array}$ & NCT02844829 & $\begin{array}{l}\text { MRI } \\
\text { Serum biomarkers } \\
\text { Urine biomarkers }\end{array}$ & Finland & 200 & July 2016 \\
\hline
\end{tabular}

Table 5. Current ongoing Phase III and IV studies investigating the diagnosis or detection of prostate cancer

\begin{tabular}{|c|c|c|c|c|c|}
\hline Study title & NCT number & Intervention & Geographic location & Number enrolled & Study start \\
\hline $\begin{array}{l}\text { PET/MRI in patients with suspected prostate } \\
\text { cancer }\end{array}$ & NCT02659527 & $\begin{array}{l}\text { Drug: }{ }^{68} \mathrm{Ga}-\mathrm{PSMA}-\mathrm{HBED}-\mathrm{CC} \text { PET } \\
\text { Device: Biograph mMR, Siemens }\end{array}$ & Austria & 220 & January 2016 \\
\hline $\begin{array}{l}{ }^{68} \mathrm{Ga}-\mathrm{PSMA} \text { PET/MRI in finding tumors in } \\
\text { patients with intermediate- or high-risk prostate } \\
\text { cancer undergoing surgery }\end{array}$ & NCT02678351 & $\begin{array}{l}\text { Drug: }{ }^{68} \mathrm{Ga}-\mathrm{PSMA} \\
\text { Procedure: MRI } \\
\text { Procedure: PET }\end{array}$ & USA & 200 & June 2016 \\
\hline${ }^{68} \mathrm{Ga}$-PSMA in preprostatectomy patients & NCT03388346 & Drug: Ga-68 PSMA-HBED-CC PET & USA & 40 & February 2018 \\
\hline${ }^{18} \mathrm{~F}-\mathrm{NaF}$ PET imaging for bone scintigraphy & NCT01930812 & $\begin{array}{l}\text { Procedure: NaF PET/CT imaging } \\
\text { Procedure: } 99 \mathrm{mTc} \text {-medronate whole } \\
\text { body bone scan with SPECT } \\
\text { Drug: }{ }^{18} \mathrm{~F} \text {-sodium fluoride }\end{array}$ & Canada & 286 & April 2014 \\
\hline $\begin{array}{l}{ }^{68} \mathrm{Ga}-\mathrm{PSM} \mathrm{M} \text { PET for patients with biochemical } \\
\text { recurrence of prostate cancer }\end{array}$ & NCT03389451 & Drug: ${ }^{68} \mathrm{Ga}-\mathrm{PSMA}-\mathrm{HBED}-\mathrm{CC}$ PET & USA & 40 & February 2018 \\
\hline $\begin{array}{l}\text { Gallium-68 PSMA- } 11 \text { PET imaging in patients } \\
\text { with biochemical recurrence }\end{array}$ & NCT03353740 & Drug: ${ }^{68} \mathrm{Ga}-\mathrm{PSMA}-\mathrm{HBED}-\mathrm{CC}$ PET & USA & 500 & October 2017 \\
\hline MRI versus PSA in prostate cancer screening & NCT02799303 & $\begin{array}{l}\text { Device: } m p M R I \\
\text { Other: PSA testing }\end{array}$ & Canada & 1010 & June 2016 \\
\hline $\begin{array}{l}\text { Staging prostate cancer with hybrid C11-choline } \\
\text { PET/MR and mpMRI }\end{array}$ & NCT03404648 & $\begin{array}{l}\text { Drug: }{ }^{11} \mathrm{C} \text { choline PET tracer } \\
\text { Drug: Gadobutrol } \\
\text { Device: PET/MR scanner }\end{array}$ & USA & 20 & November 2017 \\
\hline $\begin{array}{l}{ }^{68} \mathrm{Ga}-\mathrm{RM} 2 \mathrm{PET} / \mathrm{MRI} \text { in biochemically recurrent } \\
\text { prostate cancer }\end{array}$ & NCT02624518 & $\begin{array}{l}\text { Drug: }{ }^{68} \mathrm{Ga}-\text { labeled GRPR Antagonist } \\
\text { BAY } 86-7548 \\
\text { Procedure: MRI } \\
\text { Procedure: PET }\end{array}$ & USA & 100 & November 2015 \\
\hline $\begin{array}{l}\text { Gallium- } 68 \text { PSMA- } 11 \text { PET in intermediate- to } \\
\text { high-risk preprostatectomy patients }\end{array}$ & NCT02919111 & Drug: ${ }^{68} \mathrm{Ga}-\mathrm{PSMA}-\mathrm{HBED}-\mathrm{CC}$ PET & USA & 150 & October 2016 \\
\hline $\begin{array}{l}\text { Study of }{ }^{18} \mathrm{~F}-\mathrm{DCFPyL} \text { PET } / \mathrm{CT} \text { imaging in } \\
\text { patients with prostate cancer }\end{array}$ & NCT02981368 & Drug: ${ }^{8} \mathrm{~F}-\mathrm{DCFPyL}$ injection & USA & 377 & November 2016 \\
\hline
\end{tabular}

Identification of new molecular drug targets is key to the development of effective treatments for advanced PCa [64]. A recent study combining four profiles from the Gene Expression Omnibus identified epithelial cell adhesion molecule (EPCAM), twist family basic helix-loop-helix transcription factor 1 (TWIST1), CD38, and vascular endothelial growth factor A (VEGFA) as hub genes which may be potential therapeutic targets in PCa. Promising results have been demonstrated with agents targeting CD38 and VEGFA in the treatment of myeloma and renal carcinoma, respectively [64]. These agents may also be applied for treatment of $\mathrm{PCa}$, upon further validation of these targets and is worth investigating.

\section{Conclusions}

Early diagnosis is essential for reducing PCa mortality, due to a lack of effective treatments for advanced disease. This may be accomplished through regular PCa screening using an optimized risk predictive model. New biomarkers are continually being identified and validated, which may be used to further improve existing risk predictive models and enhance their predictive powers. Nevertheless, while 
these, as well as existing biomarkers and risk calculators require further validation, they show promise in improving the early diagnosis of PCa in Chinese men. This will assist in lowering the PCa mortality rate in China.

\section{Abbreviations}

AUC: area under the curve; CPCC-RC: Chinese Prostate Cancer Consortium Risk Calculator; cPSA: complexed prostate-specific antigen; CT: computed tomography; DRE: digital rectal examination; ERSPC-RC: European Randomized Study of Screening for Prostate Cancer Risk Calculator; FDA: Food and Drug Administration; fPSA: free prostate-specific antigen; hK2: human Kallikrein 2; MIC1: macrophage inhibitory cytokine 1; MiPS: Mi-Prostate score; mpMRI: multiparametric magnetic resonance imaging; MRF-TB: magnetic resonance imaging-ultrasound fusion targeted prostate biopsy; MSMB: microseminoprotein-beta; MSP: methylationspecific polymerase chain reaction; mSS: magnetic resonance imaging suspicion score; p2PSA: [-2]pro prostate-specific antigen; PCa: prostate cancer; PCA3: prostate cancer antigen 3; PCP: Prostate Cancer Predictor; PCPT-RC: Prostate Cancer Prevention Trial Risk Calculator; PET: positron emission tomography; PHI: Prostate Health Index; PI-RADS: Prostate Imaging Reporting and Data System; PSA: prostate-specific antigen; PSAD: prostate-specific antigen density; PV: prostate volume; ROC: receiver operating characteristic; STHLM3: Stockholm 3 study; TPM: template prostate mapping; tPSA: total prostate-specific antigen; TRUS: transrectal ultrasound.

\section{Acknowledgements}

Editorial support was provided by Joyce Lee, $\mathrm{PhD}$, of Nucleus Global, Shanghai, China, funded by AstraZeneca.

\section{Competing Interests}

The authors have declared that no competing interest exists.

\section{References}

1. Rawla P. Epidemiology of Prostate Cancer. World J Oncol. 2019; 10(2): 63-89.

2. Chen W, Zheng R, Baade PD, et al. Cancer statistics in China, 2015. CA: Cancer J Clin. 2016; 66(2): 115-32.

3. Chen WQ, Li H, Sun KX, et al. Report of cancer incidence and mortality in China, 2014. Chin J Cancer Res. 2018; 30: 1-12.

4. Siegel RL, Miller KD, Ahmedin J. Cancer statistics, 2018. CA: Cancer J Clin. 2018; 68(1): 7-30

5. Catalona WJ, Smith DS, Ratliff TL, et al. Measurement of prostate-specific antigen in serum as a screening test for prostate cancer. N Engl J Med. 1991; 324(17): 1156-61.

6. Vickers AJ, Cronin AM, Roobol MJ, et al. The relationship between prostate-specific antigen and prostate cancer risk: the Prostate Biopsy Collaborative Group. Clin Cancer Res. 2010; 16(17): 4374-81.
7. De Angelis G, Rittenhouse HG, Mikolajczyk SD, Blair Shamel L, Semjonow A. Twenty Years of PSA: From Prostate Antigen to Tumor Marker. Rev Urol. 2007; 9(3): 113-23.

8. Simmons LA, Kanthabalan A, Arya M, et al. The PICTURE study: diagnostic accuracy of multiparametric MRI in men requiring a repeat prostate biopsy. Br J Cancer. 2017; 116(9): 1159-65.

9. Ahmed HU, Bosaily AE, Brown LC, et al. Diagnostic accuracy of multi-parametric MRI and TRUS biopsy in prostate cancer (PROMIS): a paired validating confirmatory study. Lancet. 2017; 389(10071): 815-22.

10. Bussemakers MJ, van Bokhoven A, Verhaegh GW, et al. DD3: a new prostate-specific gene, highly overexpressed in prostate cancer. Cancer Res. 1999; 59(23): 5975-9.

11. Haese A, de la Taille A, Van Poppel H, et al. Clinical utility of the PCA3 urine assay in European men scheduled for repeat biopsy. Eur Urol. 2008; 54(5): 1081-8.

12. Leyten GH, Hessels D, Jannink SA, et al. Prospective multicentre evaluation of PCA3 and TMPRSS2-ERG gene fusions as diagnostic and prognostic urinary biomarkers for prostate cancer. Eur Urol. 2014; 65(3): 534-42.

13. Summary of safety and effectiveness data: PROGENSA PCA3 assay. 2012; P100033.

14. Wei JT, Feng Z, Partin AW, et al. Can urinary PCA3 supplement PSA in the early detection of prostate cancer? J Clin Oncol. 2014; 32(36): 4066-72.

15. Wang FB, Chen R, Ren SC, et al. Prostate cancer antigen 3 moderately improves diagnostic accuracy in Chinese patients undergoing first prostate biopsy. Asian J Androl. 2017; 19(2): 238-43.

16. Seisen T, Rouprêt M, Brault D, et al. Accuracy of the prostate health index versus the urinary prostate cancer antigen 3 score to predict overall and significant prostate cancer at initial biopsy. Prostate. 2015; 75(1): 103-11.

17. Guazzoni G, Nava L, Lazzeri M, et al. Prostate-specific antigen (PSA) isoform p2PSA significantly improves the prediction of prostate cancer at initial extended prostate biopsies in patients with total PSA between 2.0 and 10 $\mathrm{ng} / \mathrm{ml}$ : results of a prospective study in a clinical setting. Eur Urol. 2011; 60(2): 214-22.

18. Ferro $M$, Bruzzese $D$, Perdonà $S$, et al. Predicting prostate biopsy outcome: prostate health index (phi) and prostate cancer antigen 3 (PCA3) are useful biomarkers. Clin Chim Acta. 2012; 413(15-16): 1274-8.

19. Lazzeri M, Lughezzani G, Haese A, et al. Clinical performance of prostate health index in men with tPSA $>10 \mathrm{ng} / \mathrm{ml}$ : Results from a multicentric European study. Urol Oncol. 2016; 34(9): 415. e13,415. e19.

20. Chiu PK, Roobol MJ, Teoh JY, et al. Prostate health index (PHI) and prostate-specific antigen (PSA) predictive models for prostate cancer in the Chinese population and the role of digital rectal examination-estimated prostate volume. Int Urol Nephrol. 2016; 48(10): 1631-7.

21. Gutierrez A. P090026 Access Hybritech p2PSA on the Access Immunoassay Systems. Food and Drug Administration. 2012.

22. Parekh DJ, Punnen S, Sjoberg DD, et al. A multi-institutional prospective trial in the USA confirms that the 4Kscore accurately identifies men with high-grade prostate cancer. Eur Urol. 2015; 68(3): 464-70.

23. Punnen S, Pavan N, Parekh DJ. Finding the Wolf in Sheep's Clothing: The 4Kscore Is a Novel Blood Test That Can Accurately Identify the Risk of Aggressive Prostate Cancer. Rev Urol. 2015; 17(1): 3-13.

24. Stattin P, Vickers AJ, Sjoberg DD, et al. Improving the specificity of screening for lethal prostate cancer using prostate-specific antigen and a panel of kallikrein markers: a nested case-control study. Eur Urol. 2015; 68(2): 207-13.

25. Sjoberg DD, Vickers AJ, Assel M, et al. Twenty-year Risk of Prostate Cancer Death by Midlife Prostate-specific Antigen and a Panel of Four Kallikrein Markers in a Large Population-based Cohort of Healthy Men. Eur Urol. 2018; 73(6): 941-8.

26. Tomlins SA, Rhodes DR, Perner S, et al. Recurrent fusion of TMPRSS2 and ETS transcription factor genes in prostate cancer. Science. 2005; 310(5748): 644-8.

27. Laxman B, Tomlins SA, Mehra R, et al. Noninvasive detection of TMPRSS2: ERG fusion transcripts in the urine of men with prostate cancer. Neoplasia. 2006; 8(10): 885-8.

28. Tomlins SA, Aubin SM, Siddiqui J, et al. Urine TMPRSS2: ERG fusion transcript stratifies prostate cancer risk in men with elevated serum PSA. Sci Transl Med. 2011; 3(94): 94ra72.

29. Xue L, Mao X, Ren G, et al. Chinese and Western prostate cancers show alternate pathogenetic pathways in association with ERG status. Am J Cancer Res. 2012; 2(6): 736-44.

30. Van Neste L, Hendriks RJ, Dijkstra S, et al. Detection of High-grade Prostate Cancer Using a Urinary Molecular Biomarker-Based Risk Score. Eur Urol. 2016; 70(5): 740-8.

31. [Internet] SelectMDx for Prostate Cancer. Accessed April 7, 2019. https://mdxhealth.com/selectmdx-prostate-cancer.

32. Slaughter DP, Southwick HW, Smejkal W. "Field cancerization" in oral stratified squamous epithelium. Clinical implications of multicentric origin. Cancer. 1953; 6(5): 963-8.

33. [Internet] ConfirmMDx for Prostate Cancer. Accessed April 8, 2019. https://mdxhealth.com/confirmmdx-prostate-cancer.

34. Moldovan PC, Van den Broeck T, Sylvester R, et al. What Is the Negative Predictive Value of Multiparametric Magnetic Resonance Imaging in Excluding Prostate Cancer at Biopsy? A Systematic Review and Meta-analysis from the European Association of Urology Prostate Cancer Guidelines Panel. Eur Urol. 2017; 72(2): 250-66. 
35. Meng X, Rosenkrantz AB, Mendhiratta N, et al. Relationship Between Prebiopsy Multiparametric Magnetic Resonance Imaging (MRI), Biopsy Indication, and MRI-ultrasound Fusion-targeted Prostate Biopsy Outcomes. Eur Urol. 2016; 69(3): 512-7.

36. Kasivisvanathan V, Rannikko AS, Borghi M, et al. MRI-targeted or standard biopsy for prostate-cancer diagnosis. N Engl J Med. 2018; 378(19): 1767-77.

37. [Internet] Ankerst DP, Goros MW, Hoefler J. PCPT Risk Calculator. Accessed April 5, 2019. http://myprostatecancerrisk.com/.

38. Parekh DJ, Ankerst DP, Higgins BA, et al. External validation of the Prostate Cancer Prevention Trial risk calculator in a screened population. Urology. 2006; 68(6): 1152-5.

39. Ankerst DP, Hoefler J, Bock S, et al. Prostate Cancer Prevention Trial risk calculator 2.0 for the prediction of low- vs high-grade prostate cancer. Urology. 2014; 83(6): 1362-7.

40. [Internet] Roobol M. The Prostate Cancer Risk Calculators. Accessed April 4, 2019. http://www.prostatecancer-riskcalculator.com/seven-prostate-cancerrisk-calculators.

41. Schröder FH, Hugosson J, Roobol MJ, et al. Screening and prostate-cancer mortality in a randomized European study. N Engl J Med. 2009; 360(13): 1320-8.

42. Foley RW, Maweni RM, Gorman L, et al. European Randomised Study of Screening for Prostate Cancer (ERSPC) risk calculators significantly outperform the Prostate Cancer Prevention Trial (PCPT) 2.0 in the prediction of prostate cancer: a multi-institutional study. BJU Int. 2016; 118(5): 706-13.

43. Poyet C, Nieboer D, Bhindi B, et al. Prostate cancer risk prediction using the novel versions of the European Randomised Study for Screening of Prostate Cancer (ERSPC) and Prostate Cancer Prevention Trial (PCPT) risk calculators: independent validation and comparison in a contemporary European cohort. BJU Int. 2016; 117(3): 401-8.

44. Loeb S, Shin SS, Broyles DL, et al. Prostate Health Index improves multivariable risk prediction of aggressive prostate cancer. BJU Int. 2017; 120(1): 61-8.

45. Grönberg H, Adolfsson J, Aly M, et al. Prostate cancer screening in men aged 50-69 years (STHLM3): a prospective population-based diagnostic study. Lancet Oncol. 2015; 16(16): 1667-76.

46. Ström P, Nordström T, Grönberg H, Eklund M. The Stockholm-3 Model for Prostate Cancer Detection: Algorithm Update, Biomarker Contribution, and Reflex Test Potential. Eur Urol. 2018; 74(1): e9.

47. Eklund M, Nordström T, Aly M, et al. The Stockholm-3 (STHLM3) model can improve prostate cancer diagnostics in men aged 50-69 $\mathrm{yr}$ compared with current prostate cancer testing. Eur Urol Focus. 2016; 4(5): 707-10.

48. Tomlins SA, Day JR, Lonigro RJ, et al. Urine TMPRSS2: ERG plus PCA3 for individualized prostate cancer risk assessment. Eur Urol. 2016; 70(1): 45-53.

49. Wang Q, Li YF, Jiang J, Zhang Y, Liu XD, Li K. The establishment and evaluation of a new model for the prediction of prostate cancer. Medicine (Baltimore). 2017; 96(11): e6138.

50. Kim EH, Andriole GL. Prostate-specific antigen-based screening: controversy and guidelines. BMC Med. 2015; 13(1): 61.

51. Radtke JP, Wiesenfarth M, Kesch C, et al. Combined Clinical Parameters and Multiparametric Magnetic Resonance Imaging for Advanced Risk Modeling of Prostate Cancer-Patient-tailored Risk Stratification Can Reduce Unnecessary Biopsies. Eur Urol. 2017; 72(6): 888-96.

52. van Leeuwen PJ, Hayen A, Thompson JE, et al. A multiparametric magnetic resonance imaging-based risk model to determine the risk of significant prostate cancer prior to biopsy. BJU Int. 2017; 120(6): 774-81.

53. Wang H, Tai S, Zhang L, Zhou J, Liang C. A calculator based on prostate imaging reporting and data system version 2 (PI-RADS V2) is a promising prostate cancer predictor. Sci Rep. 2019; 9(1): 6870.

54. Sciarra A, Panebianco V, Cattarino $S$, et al. Multiparametric magnetic resonance imaging of the prostate can improve the predictive value of the urinary prostate cancer antigen 3 test in patients with elevated prostatespecific antigen levels and a previous negative biopsy. BJU Int. 2012; 110(11): 1661-5.

55. Porpiglia F, Russo F, Manfredi M, et al. The roles of multiparametric magnetic resonance imaging, PCA3 and Prostate Health Index-which is the best predictor of prostate cancer after a negative biopsy? J Urol. 2014; 192(1): 60-6.

56. Gnanapragasam VJ, Burling K, George A, et al. The Prostate Health Index adds predictive value to multi-parametric MRI in detecting significant prostate cancers in a repeat biopsy population. Sci Rep. 2016; 6: 35364.

57. Cao D, Ye D, Zhang H, Zhu Y, Wang Y, Yao X. A multiplex model of combining gene-based, protein-based, and metabolite-based with positive and negative markers in urine for the early diagnosis of prostate cancer. Prostate. 2011; 71(7): 700-10.

58. Zhu Y, Han C, Zhang G, et al. Development and external validation of a prostate health index-based nomogram for predicting prostate cancer. Sci Rep. 2015; 5: 15341.

59. Wu YS, Zhang N, Liu SH, et al. The Huashan risk calculators performed better in prediction of prostate cancer in Chinese population: a training study followed by a validation study. Asian J Androl. 2016; 18(6): 925-9.

60. Chen R, Xie L, Xue W, et al. Development and external multicenter validation of Chinese Prostate Cancer Consortium prostate cancer risk calculator for initial prostate biopsy. Urol Oncol. 2016; 34(9): 416. e1,416. e7.

61. Niu X, Li J, Das SK, Xiong Y, Yang C, Peng T. Developing a nomogram based on multiparametric magnetic resonance imaging for forecasting high-grade prostate cancer to reduce unnecessary biopsies within the prostate-specific antigen gray zone. BMC Med Imaging. 2017; 17(1): 11

62. Afef L, Rania T, Hanen C, Lamia S, Ahmed BH. Comparison study for computer assisted detection and diagnosis 'CAD'systems dedicated to prostate cancer detection using MRImp modalities. 4th International Conference on Advanced Technologies for Signal and Image Processing (ATSIP); 21 March 2018; Sousse, Tunisia. Institute of Electrical and Electronics Engineers; 2018

63. Roethke MC, Kuru TH, Mueller-Wolf M, et al. Evaluation of an Automated Analysis Tool for Prostate Cancer Prediction Using Multiparametric Magnetic Resonance Imaging. PLoS One. 2016; 11(7): e0159803.

64. Lu W, Ding Z. Identification of key genes in prostate cancer gene expression profile by bioinformatics. Andrologia. 2019; 51(1): e13169. 